\section{Unintentional Unfair Behavior Promotes Charitable Donation}

\author{
Yohsuke Ohtsubo*, Esuka Watanabe \\ Department of Psychology, Graduate School of Humanities, Kobe \\ University \\ 1-1 Rokkodai-cho, Nada, Kobe, 657-8501, Japan \\ *Author for correspondence gohtsubo@lit.kobe-u.ac.ip
}

Recent studies have demonstrated that people inflict self-punishment after unintentionally making an unfair allocation decision. The present study examined whether or not the unfair allocation decision would also prompt participants to make a charitable donation. The results indicated that participants who unintentionally made an unfair allocation decision donated a greater amount of money than those who made a fair allocation decision. In addition, the amount of money that participants donated was positively correlated with their sense of guilt ("zaiakukan" in Japanese) caused by their unfair allocation. Therefore, these results suggest that those who feel guilty (or possibly shame) due to their unintentional transgression would use charitable donation as a means to alleviate the aversive feeling.

\section{Keywords}

costly signaling theory, charitable donation, guilt, shame, dictator game

\section{Introduction}

Recent studies have demonstrated that people inflict punishment on themselves after committing some transgression, even when the transgression was unintentional (Nelissen, 2012; Nelissen \& Zeelenberg, 2009; Watanabe \& Ohtsubo, 2012). In Watanabe and Ohtsubo's (2012) experiment, all participants played a modified version of the dictator game, in which participants unintentionally made their allocation decision by drawing a card from nine cards that were placed face-down. As a result of the card-drawing, all participants made an unfair allocation. Participants were then given a chance to apologize to their partner (the costly apology condition) or privately reduce their reward (the self-punishment condition). In both conditions, approximately half of the participants were willing to make a costly apology or inflict self-punishment.

Although Ohtsubo and Watanabe (2009; see also Nelissen, 2012) consider that post-transgression costly signals (i.e., costly apology and selfpunishment) are directed toward a victim in order to communicate the transgressor's benign intention, the aforementioned result is incongruent with this interpretation. If the target of self-punishment is a victim, there is no point for participants to engage in private self-punishment, which would not be observed by the victim. Based on their experimental results, Watanabe and Ohtsubo (2012) argue that people engage in self-punishment in order to communicate their benign intention (i.e., their intention to comply with social norms) not only to their victim but also to other potential observers (i.e., other members in the same community/social group).

There is a possible criticism against Watanabe and Ohtsubo's (2012) experimental result. In assessing participants' willingness to inflict selfpunishment, Watanabe and Ohtsubo presented participants with the following probing statement: "If you are dissatisfied with the allocation you made, you can reduce your monetary reward." Given this measure of self-punishment, one might suspect that the observed self-punishment was an experimental artifact (i.e., demand characteristics). In other words, participants might have interpreted the probe as a request. Hence, it is desirable if the self-punishment would be measured in a more subtle manner. Accordingly, the present experiment employed a request for a charitable donation as a measure of self-punishment: After making an unintentional unfair or fair allocation decision, the experimenter solicited a charitable donation from participants, emphasizing that the donation was not a part of the experiment. The primary purpose of the present experiment was to test the following hypothesis.

Hypothesis 1: Participants who made an unfair allocation decision will donate a greater amount of money than those who made a fair allocation decision.

It has been well documented that the sense of guilt promotes prosocial behavior, including donations (Carlson \& Miller, 1987; Salovey, Mayer, \& Rosenhan, 1991, for reviews). For example, in Darlington and Macker's (1966) study, participants were led to believe that whether or not their partner would receive an experimental reward (i.e., course credit) would be determined by how well they performed an experimental task. Half of participants received the success feedback, while the other half received the failure feedback. After the task, a different experimenter asked participants for a blood donation. Those who failed the task (thus failed to help their partner earn the extra credit) were more likely to donate their blood than those 
who succeeded. In a more recent experiment (Sachdeva, Iliev, \& Medin, 2009), participants who were reminded of their negative aspects (e.g., greediness, selfishness) donated a greater amount of money to a charity of their choice than participants who were reminded of their positive aspects (e.g., generosity, kindness).

Although these findings suggest that participants' donating behavior was mediated by their sense of guilt, neither Darlington and Macker's (1966) study nor Sachdeva et al.'s (2009) study directly assessed participants' emotional state. Thus, the second purpose of the present study was to directly test whether self-punishment measured by charitable donation would be predicted by participants' self-reported guilt.

Hypothesis 2: Participants who feel a stronger sense of guilt will donate a greater amount of money.

Recall that Watanabe and Ohtsubo (2012) observed the significant positive correlation between self-punishment and self-reported guilt. Therefore, if the donating behavior in the present study is positively correlated with the self-reported guilt, it supports the notion that guilty people would use a charitable donation as a form of self-punishment.

\section{Method}

(a) Participants and Design

Participants were 102 Japanese undergraduates (mean age $\pm \mathrm{SD}=18.95 \pm 1.02$ ) who voluntarily took part in the experiment in exchange for some monetary reward. Participants were informed that they would be guaranteed to earn 500 Japanese yen (JPY) as a show-up fee and an additional unspecified amount of money from participating in an experimental game. They were randomly assigned to either the fair allocation condition or the unfair allocation condition.

\section{(b) Procedure}

Participants played the modified version of the dictator game used in Watanabe and Ohtsubo's (2012) study. Participants were asked to allocate 500 JPY between themselves and a partner. As an allocator, participants drew a card from nine cards that were placed face-down on a desk. Although participants were led to believe that the nine cards were associated with various allocation schemes, participants in fact made either a fair allocation decision (i.e., $250 \mathrm{JPY}$ to each) or an unfair allocation decision (i.e., $400 \mathrm{JPY}$ to themselves and 100 JPY to their partner).

After completing this allocation task, participants filled out a questionnaire that apparently aimed to assess various aspects of the allocation task. Although the guilt item was embedded in this questionnaire, it made no sense to ask participants in the fair allocation condition to indicate their sense of guilt. Accordingly, only those who were assigned to the unfair allocation condition were asked to indicate their sense of guilt ("zaiakukan" in Japanese) on a 5-point scale ( $1=$ "not at all" to $5=$ "very much"). This item was equivalent to the measure used in Watanabe and Ohtsubo's (2012) Experiment 2.

The experimenter (E) then declared that the experiment was over. Next, E explained that in an attempt to contribute to society, her laboratory asks participants to make a donation. E gave participants a sheet of paper listing the names of four actual charity organizations. Participants indicated the amount of money they were willing to donate, and the name of one organization to which they would like to donate. The sheet explicitly instructed participants to write " $0 \mathrm{JPY}$ " when they did not wish to donate their money. After completing this donation decision, E thoroughly debriefed and paid 1000 JPY to all the participants.

\section{Results}

Two participants who suspected the presence of deceptive procedures were omitted from the subsequent data analyses. As shown in Figure 1 , participants were willing to donate a greater amount of money in the unfair allocation condition $(60.00 \pm 71.85, \mathrm{n}=54)$ than in the fair allocation condition $(44.13 \pm 84.08, \mathrm{n}=46)$. Since the donation data were not normally distributed (according to the Shapiro-Wilk test, its distribution significantly deviated from the normal distribution, $\mathrm{p}<.001$ ), a non-parametric test was used to test Hypothesis 1. The Mann-Whitney U test indicated that the difference was significant, $\mathrm{z}=2.02, \mathrm{p}=.044$. Therefore, Hypothesis 1 was supported.

We then tested whether donation was predicted by participants' reported sense of guilt ("zaiakukan" in Japanese). Since we did not include the measure

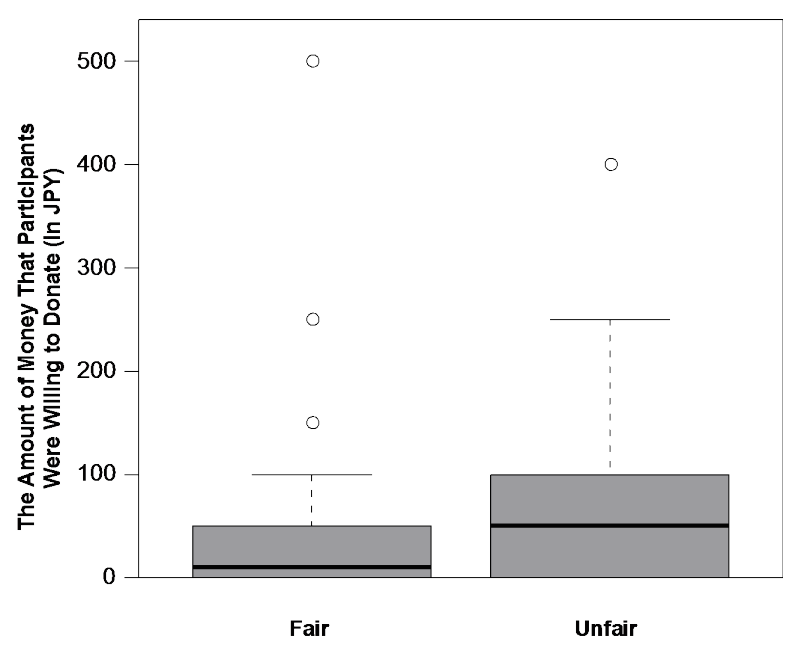

Figure 1. The distribution of the amount of money that participants were willing to donate as a function of the allocation fairness condition. 
of guilt in the fair allocation condition, the subsequent analyses only included the participants in the unfair allocation condition. Participants who reported a higher level of guilt were willing to donate a greater amount of money, $\mathrm{r}(51)=.34, \mathrm{p}=$ .011. Since the donation data were positively skewed (skewness $=3.79$ in the unfair allocation condition), the comparable correlation was computed for the square-root transformed data. The correlation was again significant, $\mathrm{r}(51)=.37, \mathrm{p}=.007$. Therefore, Hypothesis 2 was supported.

Participants in the present study completed the Test of Self-Conscious Affect(TOSCA; Tangney \& Dearing, 2002) approximately one week after the donation experiment. Therefore, each participant's disposition to experience shame and guilt was identifiable. For an exploratory purpose, whether participants' predisposition to experience guilt and shame (i.e., trait guilt and trait shame) would predict their state guilt after an unfair allocation decision was tested. The reported guilt ("zaiakukan") in the donation experiment was significantly correlated with participants' trait shame, $\mathrm{r}(51)=.33, \mathrm{p}=.014$, but not with their trait guilt, $\mathrm{r}(51)=.18$, ns. Although the difference between the correlation coefficients was not statistically significant, $\mathrm{z}=0.81 . \mathrm{ns}$, the result suggests that the sense of "zaiakukan" in the present study was more strongly related to shame than to guilt.

\section{Discussion}

The present experiment demonstrated that unintentional unfair allocation would promote charitable donations. Participants who made an unfair allocation decision subsequently donated a greater amount of money than those who made a fair allocation decision. Among the participants who made the unfair allocation, their sense of guilt (which might possibly be a sense of shame) was correlated with the amount of money they were willing to donate. These two findings jointly support the notion that a transgression promotes compensatory prosocial behavior, and this effect is mediated by aversive feelings engendered by committing a transgression.

There is evidence that charitable donations confer reputational as well as material benefits on the donors. Donors are more likely to be perceived as being more generous, to become popular as an interaction partner, and to receive income transfers from members of their group (Fehrler \& Przepiorka, in press; Milinski, Semmann, \& Krambeck, 2002). In addition, public participation in charitable activities and blood donations seem to have a similar positive reputation effect (Bereczkei, Birkas, \& Kerekes, 2010; Lyle, Smith, \& Sullivan, 2009). Therefore, charitable donations can be conceived as a form of costly signals of signaler quality (e.g., trustworthiness, physical fitness). The present study provided additional evidence that donations can be used as a means to maintain signalers' reputation when benign signalers inadvertently committed a transgression. In such circumstances, donations can be used to advertise signalers' benign intent to comply with social norms in the future.

Although it was not reported in the result section, effect size was greater for male participants than for female participants. Cohen's d's were .54 and .27 for males and females, respectively (for the female sample, an outlier participant who donated 500 JPY in the fair allocation condition was omitted). When the Mann-Whitney U test was computed separately for males and females, the effect was significant for males $(p=.03)$, but not significant for females $(p=.37)$. This might be due to a significantly higher base donation rate in the fair condition: females donated a greater amount of money $(40.87 \pm 40.67)$ than males $(26.82 \pm 56.52), \mathrm{p}=$ .049 by the Mann-Whitney U test. Since previous research found no sex difference regarding selfpunishment tendency, further research is needed to draw any strong conclusion.

An unexpected result of the present study was that the sense of "zaiakukan" was more strongly correlated with trait shame than with trait guilt. Therefore, although "zaiakukan" is typically considered to be the Japanese equivalence of "guilt," the present study indicated that Japanese "zaiakukan" also refers to a sense of shame. This result implies that state shame, not state guilt, is a proximate cause of self-punishment. Nonetheless, in the present study, neither trait shame nor trait guilt was significantly correlated with donation decision, r's $=.13$ and -.03 for trait shame and trait guilt, respectively. Notice that participants' donation decision was determined by multiple factors other than their sense of guilt/shame. For example, it is plausible that more altruistic participants donated more money than less altruistic participants. Whether participants' trait shame and trait guilt would predict self-punishment might be better tested by using Watanabe and Ohtsubo's (2012) original assessment, a pure form of selfpunishment, that is not likely to be facilitated by factors other than aversive feelings.

\section{Acknowledgements}

This research was supported by the Japan Society for the Promotion of Science (No. 21683006). The authors are grateful to Asami Matsumura for her assistance in conducting the experiment.

\section{References}

Bereczkei, T., Birkas, B., \& Kerekes, Z. (2010). Altruism towards strangers in need: costly signaling in an industrial society. Evolution and Human Behavior, 31, 95-103. doi: 10.1016 i.evolhumbehav.2009.07.004)

Carlson, M., \& Miller, N. (1987). Explanation of the relation between negative mood and helping. 
Psychological Bulletin, 102, 91-108. doi: 10.1037/0033-2909.102.1.91)

Darlington, R. B., \& Macker, C. E. (1966). Displacement of guilt-produced altruistic behavior. Journal of Personality and Social Psychology, 4, 442-443. doi: 10.1037/h0023743)

Fehrler, S., \& Przepiorka, W. (in press). Charitable giving as a signal of trustworthiness: Disentangling the signaling benefits of altruistic acts. Evolution and Human Behavior. doi: 10.1016/i.evolhumbehav.2012.11.005

Lyle, H. F., III, Smith, E. A., \& Sullivan, R. J. (2009). Blood donations as costly signals of donor quality. Journal of Evolutionary Psychology, 7, 263-286. doi: 10.1556/JEP.7.2009.4.1)

Milinski, M., Semmann, D., \& Krambeck, H.-J. (2002). Donors to charity gain in both indirect reciprocity and political reputation. Proceedings of the Royal Society of London B, 269, 881-883. doi: 10.1098/rspb.2002.1964

Nelissen, R. M. A. (2012). Guilt-induced self-punishment as a sign of remorse. Social Psychological and Personality Science, 3, 139-144. doi. 10.1177/1948550611411520

Nelissen, R. M. A., \& Zeelenberg, M. (2009). When guilt evokes self-punishment: Evidence for the existence of a Dobby effect. Emotion, 9, 118-122. doi: 10.1037/a0014540

Ohtsubo, Y., \& Watanabe, E. (2009). Do sincere apologies need to be costly? Test of a costly signaling model of apology. Evolution and Human Behavior, 30, 114-123. doi: 10.1016 i.evolhumbehav.2008.09.004

Sachdeva, S., Iliev, R., \& Medin, D. L. (2009). Sinning saints and saintly sinners: The paradox of moral self-regulation. Psychological Science, 20, 523-528. doi: 10.1111/i.1467-9280.2009.02326. Q)

Salovey, P. Mayer, J. D., \& Rosenhan, D. L. (1991). Mood and helping: Mood as a motivator of helping and helping as a regulator of mood. In M. S. Clark (Ed.), Prosocial behavior (pp. 215-237). Newbury Park, CA: Sage.

Tangney, J. P. \& Dearing, R. L. (2002). Shame and guilt. New York: Guilford Press.

Watanabe, E., \& Ohtsubo, Y. (2012). Costly apology and self-punishment after an unintentional transgression. Journal of Evolutionary Psychology, 10, 87-105. doi: 10.1556/JEP.10.2012.3.1 